\section{Psicología IBEROAMERICANA}

\section{Psicología Iberoamericana}

\section{ISSN: 1405-0943}

psicología.iberoamericana@uia.mx

Universidad Iberoamericana, Ciudad de

México

México

Colín Flores, Carlos Gabriel; Simón Domínguez, Nadima

Adicción al trabajo, satisfacción y desempeño laboral en ejecutivos mexicanos

Psicología Iberoamericana, vol. 22, núm. 2, julio-diciembre, 2014, pp. 16-24

Universidad Iberoamericana, Ciudad de México

Distrito Federal, México

Disponible en: http://www.redalyc.org/articulo.oa?id=133938134003

- Cómo citar el artículo

Número completo

- Más información del artículo

Página de la revista en redalyc.org

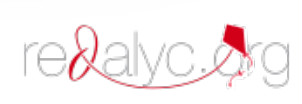

Sistema de Información Científica

Red de Revistas Científicas de América Latina, el Caribe, España y Portugal Proyecto académico sin fines de lucro, desarrollado bajo la iniciativa de acceso abierto 


\title{
Adicción al trabajo, satisfacción y desempeño laboral en ejecutivos mexicanos
}

\author{
Work Addiction, Job Satisfaction and Performance among Mexican Executives
}

\author{
Carlos Gabriel Colín Flores* \\ Nadima Simón Domínguez \\ Facultad de Psicología \\ Universidad nacional Autónoma de México
}

\section{RESUMEN}

Se reportan los resultados de un estudio de caso de una empresa multinacional mexicana, sobre la adicción al trabajo y la relación de ésta con la satisfacción y desempeño laboral en ejecutivos mexicanos. Se determinó que 28.4\% de los ejecutivos de una muestra de 74 participantes son adictos al trabajo. Asimismo, se encontró una correlación positiva entre la adicción al trabajo y factores como trabajo en exceso $r=0.93$, trabajo compulsivo $r=0.96$, factores intrínsecos de satisfacción laboral $r=0.78$, satisfacción laboral general $r=0.79$ y desempeño $r=0.31$. Además de la identificación de la correlación con otras variables como: edad, puesto de trabajo, horas trabajadas y antigüedad. Estas variables conforman un perfil del ejecutivo mexicano adicto al trabajo. Adicionalmente, se presenta el resultado de un análisis de regresión lineal, que muestra que la adicción al trabajo depende de los factores de trabajo en exceso, trabajo compulsivo y factores intrínsecos de satisfacción laboral.

Palabras clave: adicción al trabajo, satisfacción laboral, desempeño laboral, trabajo en exceso.

\section{ABSTRACT}

This research shows the results of a case study on a multinational Mexican firm, regarding work addiction and its relationship to job satisfaction and performance among Mexican executives. It was found that $28.4 \%$ of executives out of a sample of 74 participants were workaholics, and a positive correlation was found between work addiction and factors such as overwork $r=0.93$, compulsive work $r=0.96$, and factors inherently related to job satisfaction $r=0.78$, general job satisfaction $r=0.79$ and performance $r=0.31$. The correlation with other variables such as age, position, hours worked and seniority was also identified. These variables create a profile of Mexican executives addicted to work. The results of a linear regression analysis are also given, showing that addiction to work depends on factors including overwork, compulsive work, and factors inherently related to job satisfaction.

Keywords: Addiction to Work, Job Satisfaction, Job Performance, Overwork. 


\section{INTRODUCCIÓN}

En 1971, Wayne Oates publicó el primer documento formal sobre el fenómeno de adicción al trabajo, en el que definió a ésta como "una necesidad de trabajar incesantemente que afecta a la salud, a la felicidad y a las relaciones de la persona" (Oates, 1971).

Desde el planteamiento primigenio de Oates han existido diferentes ópticas sobre la definición de la adicción al trabajo: hay quienes han tenido una visión positiva y otros, una percepción negativa sobre este tipo de adicción psicológica.

La perspectiva positiva de la adicción al trabajo -con un punto de vista basado en los beneficios para la organización - tuvo su mayor predominancia en los primeros años de estudio del concepto, en autores como Machlowitz (1978); Korn, Pratt y Lambrou (1987).

En la línea del enfoque negativo de la adicción al trabajo, Fassel (1990) afirma que: "La adicción al trabajo es una enfermedad progresiva y fatal que se enmascara como un rasgo positivo en nuestra cultura". Para esta autora, los adictos se alienan de sus propios cuerpos, de sus sentimientos, de su creatividad y de su familia y amigos. Otros autores, en la misma dirección que Fassel, han apoyado sus investigaciones sobre el efecto perjudicial que tiene la adicción al trabajo en quien la padece (Killinger, 1991; Spence \& Robbins, 1992; Porter, 1996, Robinson, 1997; Snir \& Zohar, 2000; Schaufeli, Taris \& Bakker, 2006).

La predominancia de enfoques - positivo o negativo- fue estudiada por Del Líbano y Llorens (2010), empleando la base de datos Psychoinfo y encontraron que a junio de 2009, la proporción de investigaciones que consideran la adicción al trabajo como un fenómeno negativo con consecuencias perniciosas para el trabajador era de 10:1, sobre las que lo consideraban positivo.

Las consecuencias negativas de la adicción al trabajo son claras, no sólo en el ámbito individual -problemas de salud y de comunicación-, sino también a escala grupal -malas relaciones entre compañeros de trabajo y desestructuración familiar- (Del Líbano \& Llorens, 2010; Robinson, 1998).

Este trabajo se apoya en que el constructo de la adicción al trabajo es perjudicial para la persona que la sufre.
La definición de adicción al trabajo empleada en esta investigación es la siguiente: "La adicción al trabajo es un daño laboral de tipo psicosocial, que se caracteriza por el trabajo excesivo que un individuo lleva a cabo debido a una irresistible necesidad o impulso de trabajar constantemente" (Salanova, Del Líbano, Llorens, Schaufeli \& Fidalgo, 2008).

Por otra parte, tomando como referencia la presencia del fenómeno de adicción al trabajo en distintas culturas, la proporción de ejecutivos adictos al trabajo en Estados Unidos es 23.0\% de su población económicamente activa (PEA), de acuerdo con Doerfler y Kramer (1986); por otro lado, Kanai, Wakabayashi y Fling (1996) reportaron que en una muestra de más de 600 participantes en Japón, compuesta principalmente por gerentes, la cifra de adictos al trabajo fue de 21\%. En México, tomando el criterio propuesto por Mosier (1983), que considera como adicto al trabajo a la persona que trabaja más de 48 horas por semana, se estima que $28 \%$ de la PEA del país entra en el grupo de adictos al trabajo (INEGI, 2012), que es una proporción relevante de los trabajadores mexicanos.

Tanto por el aspecto perjudicial para las personas que sufren la adicción al trabajo como por la proporción de la población trabajadora que pudiera hallarse en este grupo, se consideró la importancia de hacer una investigación sobre este tema.

Por otro lado, ya que el adicto al trabajo invierte gran parte de su tiempo en sus acividades laborales, podría suponerse que se encuentra satisfecho con éstas. A raíz de tal pensamiento surge de manera casi obvia la pregunta: ¿el adicto al trabajo se encuentra satisfecho laboralmente?, para tratar de contestarla, en principio, se tiene que explicar qué es la satisfacción laboral, que Locke (1976) define como un "estado emocional positivo o placentero de la percepción subjetiva de las experiencias laborales del sujeto". Entonces, pensar que un adicto al trabajo está satisfecho laboralmente puede ser un supuesto válido y además, con base en éste, pudiera tomarse como premisa que la satisfacción laboral en el adicto al trabajo puede potenciar en él o en sus colaboradores dicha adicción.

En el presente trabajo se tomó el postulado de Herzberg (1968) como base para medir la satisfacción laboral, que establece la existencia de dos grupos de aspectos laborales: un grupo de factores extrínsecos y 
otro de factores intrínsecos. Los primeros se refieren a las condiciones de trabajo en el sentido más amplio, que Herzberg llama factores de contexto, como el salario, las políticas de empresa, el entorno físico, la seguridad en el trabajo, etc. Según el modelo bifactorial de Herzberg, estos factores extrínsecos sólo pueden prevenir la insatisfacción laboral o evitarla cuando exista, pero no pueden determinar la satisfacción, ya que ésta se encontraría determinada por los factores intrínsecos, aquellos que son inherentes al trabajo, como: el contenido de éste, los retos, la responsabilidad, el logro, entre otros. Por lo que podría suponerse que existe una relación positiva entre los factores intrínsecos de satisfacción laboral y la adicción al trabajo.

A la vez, podría considerarse que un adicto al trabajo tiene un buen desempeño laboral, ya que le satisface hacer su trabajo y disfruta cumplir con sus metas. En referencia a este término, Milkovich y Boudreau (1994), definen el desempeño laboral como el grado en el cual el empleado cumple con los requisitos de trabajo.

Con base en los conceptos de adicción al trabajo, la satisfacción y el desempeño laboral, las preguntas de investigación planteadas en este trabajo fueron: ¿cuál es la proporción de ejecutivos mexicanos que presenta adicción al trabajo en una empresa?; ¿cuáles son los factores que explican la adicción al trabajo en los ejecutivos de esta empresa?; ¿cuál es la relación entre la adicción al trabajo, la satisfacción y el desempeño laboral en los ejecutivos de la organización?

\section{MÉTODO}

En esta investigación se presenta un estudio de caso, que se llevó a cabo en una empresa mexicana que realiza operaciones multinacionales con ventas mayores a los siete mil millones de dólares por año, colaboran en ella más de setenta y cinco mil empleados, y en la oficina central cuenta con 441 ejecutivos organizados en supervisores, gerentes y directores.

\section{Participantes}

Se seleccionó una muestra estratificada y aleatoria de 95 ejecutivos mexicanos -cinco directores, 35 gerentes y 55 supervisores- de una empresa multinacional mexicana.

\section{Instrumentos}

El instrumento que se seleccionó para medir la adicción al trabajo fue el cuestionario Duwas-10 -Dutch Work Addiction Brief Scale of 10 item- propuesto y validado por Del Líbano, Llorens, Salanova y Schaufeli (2010), que se evalúa con una escala de Likert de 1 a 4 -en la que 1 es "totalmente en desacuerdo" y 4 es "totalmente de acuerdo"-, y arroja como resultado dos factores: trabajo en exceso y trabajo compulsivo -éste cuenta con un alfa de Cronbach de 0.75 en su aplicación en Holanda y 0.85 en España-. Por otra parte, para medir la satisfacción laboral general se tomó el cuestionario de Cook, Wall y Warr (1981), que cuenta con 15 reactivos y se califica con una escala de Likert de 1 a 7 -en la que 1 es "totalmente en desacuerdo" y 7 es "totalmente de acuerdo"-, y que mide los factores intrínsecos y extrínsecos de satisfacción laboral; la combinación de ambos permite medir la satisfacción laboral general -éste cuenta con un alfa de Cronbach de 0.85 en su aplicación en España-. Adicionalmente, se solicitó al área de recursos humanos de la empresa en estudio, los datos de desempeño laboral de cada participante en la muestra.

La variable dependiente fue el nivel de adicción al trabajo y las variables independientes fueron: edad, sexo, estado civil, nivel de estudios, antigüedad en la empresa, horas trabajadas en exceso, factor trabajo en exceso, factor trabajo compulsivo, factores intrínsecos, factores extrínsecos, satisfacción laboral general y desempeño laboral.

\section{Procedimiento}

Los cuestionarios fueron entregados en mano a cada ejecutivo y se les dio una semana para contestarlo, al término de ésta se recogieron 77 cuestionarios y se descartaron tres por tener respuestas incompletas, por lo que quedaron 74 cuestionarios válidos para elaborar el análisis de resultados, la tasa de no contestación quedó en $22 \%$. Se trabajó con la muestra completa y se identificó a dos subgrupos en ésta: el de los ejecutivos adictos al trabajo y el de los no adictos al trabajo. Los datos fueron procesados en spss, versión 17.0. 


\section{RESULTADOS}

Se realizó el análisis factorial exploratorio con rotación Varimax para el cuestionario Duwas-10, en el que se encontraron los dos factores propuestos por los autores: trabajo en exceso y trabajo compulsivo, en la corrida para este estudio de caso se obtuvo un alfa de Cronbach de 0.91 y una explicación de la varianza para los factores trabajo en exceso y el trabajo compulsivo de 59 y $14 \%$, respectivamente. El factor trabajo en exceso cuenta con una predominancia clara frente al de trabajo compulsivo, este último comparte algunas variables con una carga factorial significativa, con el factor trabajo en exceso ( $v i d$. tabla 1 ).
Asimismo, se realizó el análisis factorial exploratorio con rotación Varimax para el cuestionario de satisfacción laboral de Cook, Wall y Warr (1981), en el que se encontraron los dos factores propuestos por los autores: factores intrínsecos de satisfacción laboral y factores extrínsecos de satisfacción laboral, se obtuvo un alfa de Cronbach de 0.96 y una explicación de la varianza para los factores intrínsecos y extrínsecos de satisfacción laboral de 67 y 8\%, respectivamente. En este caso, los factores intrínsecos de satisfacción son dominantes en el análisis (vid. tabla 2).

Tabla 1. Ponderaciones de factores para el análisis factorial exploratorio con rotación Varimax de las escalas de adicción al trabajo. Escala Duwas-10.

\begin{tabular}{|c|c|c|}
\hline Escala & Trabajo en exceso & Trabajo compulsivo \\
\hline Me siento obligado a trabajar, incluso cuando no lo disfruto. & 0.885 & 0.006 \\
\hline Me resulta difícil relajarme cuando tengo un día libre. & 0.861 & 0.269 \\
\hline Dedico más tiempo a trabajar que a estar con mis amigos. & 0.774 & 0.442 \\
\hline Parece que estoy contra el reloj. & 0.724 & 0.433 \\
\hline Me siento culpable cuando tengo un día libre. & 0.719 & 0.505 \\
\hline $\begin{array}{l}\text { Me encuentro trabajando continuamente, después de que todos mis } \\
\text { compañeros se han ido. }\end{array}$ & 0.708 & 0.558 \\
\hline Generalmente estoy ocupado y tengo muchos asuntos entre manos. & 0.672 & 0.432 \\
\hline Me encuentro a mí mismo haciendo dos o tres cosas al mismo tiempo. & 0.617 & -0.197 \\
\hline Para mí es importante trabajar duro aun cuando no lo disfruto. & 0.287 & 0.885 \\
\hline Siento que hay algo dentro de mí que me impulsa a trabajar duro. & 0.043 & 0.84 \\
\hline $\begin{array}{r}\text { Extracción de la suma de cuadrados. } \\
\text { Valores eigen. } \\
\% \text { de varianza. }\end{array}$ & $\begin{array}{c}5.897 \\
58.973\end{array}$ & $\begin{array}{c}1.422 \\
14.222\end{array}$ \\
\hline
\end{tabular}

Nota: Factores de ponderación $>0.40$ en negritas.

Fuente: elaborado con base en resultados del análisis factorial utilizando sPss statistics 17.0 
Tabla 2. Ponderaciones de factores para el análisis factorial exploratorio con rotación Varimax de las escalas de adicción al trabajo. Escala de satisfacción laboral general (Cook, Wall y Warr, 1981).

\begin{tabular}{|c|c|c|}
\hline Escala & Factores Intrínsecos & Factores Extrínsecos \\
\hline Relación entre dirección y trabajadores. & 0.910 & 0.189 \\
\hline Posibilidades de promoción. & 0.907 & 0.209 \\
\hline Variedad de las tareas que haces. & 0.885 & 0.272 \\
\hline Salario. & 0.869 & 0.279 \\
\hline Responsabilidad asignada. & 0.863 & 0.336 \\
\hline Horario de trabajo. & 0.846 & 0.195 \\
\hline Atención a las sugerencias hechas. & 0.826 & 0.391 \\
\hline Posibilidad de utilizar capacidades personales. & 0.782 & 0.364 \\
\hline Estabilidad en el empleo. & 0.763 & 0.155 \\
\hline Modo de gestión de la empresa. & 0.726 & 0.441 \\
\hline Libertad para elegir tu propio método de trabajo. & 0.642 & 0.375 \\
\hline Tu superior inmediato. & 0.610 & 0.591 \\
\hline Reconocimiento que obtienes por el trabajo bien hecho. & 0.560 & 0.588 \\
\hline Tus compañeros de trabajo. & 0.083 & 0.806 \\
\hline Condiciones físicas del trabajo. & 0.240 & 0.779 \\
\hline $\begin{array}{r}\text { Extracción de la suma de cuadrados. } \\
\text { Valores eigen. } \\
\% \text { de varianza. }\end{array}$ & $\begin{array}{l}10.053 \\
67.022\end{array}$ & $\begin{array}{l}1.202 \\
8.010\end{array}$ \\
\hline
\end{tabular}

Nota: Factores de ponderación $>0.40$ en negritas.

Fuente: elaborado con base en resultados del análisis factorial utilizando sPSS statistics 17.0

En el cuestionario de satisfacción laboral, la variable de la escala "relación entre dirección y trabajadores" presenta una ponderación alta en el análisis factorial, lo que puede interpretarse como la importancia que tiene la influencia de las personas clave en la organización; otra variable destacada de la escala es "posibilidades de promoción", relacionada con la expectativa del ejecutivo sobre su crecimiento en la organización; ambas variables influyen en la satisfacción laboral en esta empresa.

A su vez, el análisis descriptivo de la muestra completa -74 participantes- indica que $85 \%$ de los ejecutivos están en un rango de edad entre 20 y 40 años. En cuanto a sexo, son hombres $70.3 \%$. Respecto al nivel educativo, $71.6 \%$ de los participantes tiene estudios profesionales y $23.0 \%$ cuenta con posgrado. Sobre su estado civil, $48.6 \%$ corresponde a solteros, $32.4 \%$ casados y $18.9 \%$ divorciados. En relación con el cargo de los ejecutivos representados en la muestra, $60.8 \%$ es de supervisores, $33.8 \%$ gerentes y $5.4 \%$ directores. El tiempo trabajado al día por los ejecutivos, en promedio, fue de 12.4 horas. Tienen más de cuatro años en la organización $40.5 \%$ de los ejecutivos, con un promedio de 4.5 años.

De acuerdo con los resultados del cuestionario Duwas-10 y los criterios de evaluación establecidos 
por los autores de éste, $28.4 \%$ de los ejecutivos es adicto al trabajo y $71.6 \%$ no lo es.

En relación con la satisfacción laboral, 50\% de los ejecutivos en la muestra está moderadamente satisfecho y $11 \%$ está satisfecho. Respecto al desempeño de los ejecutivos, el promedio fue de $82 \%$ de cumplimiento de los objetivos.

En referencia a la correlación entre adicción al trabajo y las variables independientes, las que muestran un coeficiente de correlación de Spearman significativo a un nivel $p<0.01$ para la muestra completa, son: edad $r=0.412$, horas trabajadas en exceso $r=0.596$, antigüedad $r=0.513$, trabajo en exceso $r=0.933$, trabajo compulsivo $r=0.964$, factores intrínsecos de satisfacción laboral $\mathrm{r}=0.775$, factores extrínsecos de satisfacción laboral $\mathrm{r}=0.780$, satisfacción laboral general $\mathrm{r}=0.789$, y desempeño $\mathrm{r}=0.314$.

Las correlaciones significativas con un $p<0.01$ (dos colas), entre adicción al trabajo y las variables in- dependientes que se presentan de manera consistente tanto en la muestra completa $(\mathrm{N}=74)$ como en el subgrupo de adictos al trabajo $(\mathrm{n}=21)$ y el subgrupo de no adictos al trabajo $(n=53)$, son: trabajo en exceso, trabajo compulsivo, factores intrínsecos y satisfacción general laboral (vid. tabla 3 ).

Además, para corroborar la importancia de las variables identificadas como relevantes en el análisis de correlación, se realizó el análisis de regresión lineal para dichas variables, que dio como resultado la ecuación:

\section{Adicción_Trabajo $=4.423+1.14 *$ T_Exceso + $0.76{ }^{\star} \mathrm{T}$ _Compulsivo $-0.047{ }^{\star} \mathrm{F} \_$Intrínsecos}

Esta ecuación presentó una $\mathrm{R}^{2}$ de 0.83 , con un viF de 1.15, la prueba de Durbin Watson tuvo un valor de 1.83 y una media de residuales de cero, parámetros que indican ausencia de colinealidad.

Tabla 3. Resultados de los coeficientes de correlación de Spearman

\begin{tabular}{|c|c|c|c|}
\hline \multirow[t]{2}{*}{ Variable independiente } & \multicolumn{3}{|c|}{ Variable dependiente (adicción al trabajo) } \\
\hline & $\begin{array}{l}\text { Total de la muestra } \\
\qquad N=74\end{array}$ & $\begin{array}{l}\text { Adictos al trabajo } \\
\qquad n=21\end{array}$ & $\begin{array}{l}\text { No adictos al trabajo } \\
n=53\end{array}$ \\
\hline Edad & $0.412 * *$ & $0.469 *$ & 0.004 \\
\hline Sexo & 0.125 & -0.309 & $0.317^{*}$ \\
\hline Educación & -0.035 & 0.284 & 0.206 \\
\hline Estado civil & -0.093 & 0.307 & $-0.396 * *$ \\
\hline Puesto de trabajo & $-0.352 *$ & $-0.698 * *$ & -0.056 \\
\hline Horas trabajadas en exceso & $0.596 * *$ & -0.040 & 0.021 \\
\hline Antigüedad & $0.513^{* *}$ & $0.536^{* *}$ & 0.116 \\
\hline Trabajo en exceso & $0.933^{* *}$ & $0.848^{* *}$ & $0.822 * *$ \\
\hline Trabajo compulsivo & $0.964 * *$ & $0.879 * *$ & $0.907 * *$ \\
\hline Factores intrínsecos & $0.775^{* *}$ & $0.932 * *$ & $0.421 * *$ \\
\hline Factores extrínsecos & $0.780 * *$ & 0.257 & $0.445^{* *}$ \\
\hline Satisfacción laboral general & $0.789 * *$ & $0.949 * *$ & $0.432 * *$ \\
\hline Desempeño & $0.314 * *$ & $0.692 * *$ & 0.101 \\
\hline
\end{tabular}

** Correlación significativa al nivel $p<0.01$ ( 2 colas)

* Correlación significativa al nivel $p<0.05$ (2 colas)

Fuente: elaborado con base en resultados del análisis factorial utilizando spss statistics 17.0 


\section{DISCUSIÓN DE RESULTADOS}

En el análisis factorial exploratorio del cuestionario Duwas-10, se identificó que el factor con mayor importancia para medir la adicción al trabajo es el de trabajo en exceso -aunque el factor trabajo compulsivo presenta una explicación de la varianza relativamente baja, las cargas factoriales en algunas variables de la escala se encuentran combinadas con ponderaciones relevantes de las variables de la escala de trabajo en exceso, por lo que no se descarta como un factor que explica la adicción al trabajo-; por otra parte, en el cuestionario de satisfacción laboral (Cook, Warr \& Wall, 1981), el factor dominante es el de factores intrínsecos de satisfacción laboral. Estas tres variables -trabajo en exceso, trabajo compulsivo y factores intrínsecos-, explican la adicción al trabajo; situación que se aprecia en el análisis de regresión lineal y el análisis de correlación.

Asimismo, como se esperaba, se encontró una magnitud relevante en la proporción de adictos al trabajo en el estudio de caso, que se ubicó en $28.4 \%$ de los ejecutivos de la muestra. La cifra hallada en este estudio coincide con la proporción de la población económicamente activa mexicana que trabaja más de 48 horas (INEGI, 2012).

Respecto a la correlación entre el nivel de adicción al trabajo y la variable posición jerárquica $(\mathrm{r}=0.35)$, asociada con la variable de la escala de satisfacción laboral, relación entre la dirección y trabajador -que presenta la mayor carga factorial-, deriva la conclusión de que hay un estilo de liderazgo jerárquico en la organización, donde las personas clave como directores y gerentes que, en su mayoría, son adictos al trabajo e influyen en el quehacer diario de la empresa, pueden fomentar comportamientos adictivos al trabajo en los colaboradores, ya que las reglas del juego las ponen las personas clave y las siguen los colaboradores, con el afán de crear una buena relación entre dirección y empleados en la organización. A manera de ejemplo de esa situación, los empleados que más trabajan en la empresa sujeta a estudio son los adictos al trabajo, y son también las personas clave -trabajan más de 14 horas diarias-. En sintonía con esta cultura de las personas clave que trabajan por largas jornadas, los empleados no adictos al trabajo también muestran un horario de trabajo extenso, promediando 12.5 horas diarias; es decir, 4.5 horas más de lo que establece su horario de contrato. En la misma dirección de lo observado en este estudio, Porter (2006) afirma que en sus investigaciones encontró que es frecuente que en las organizaciones se fomente la adicción al trabajo, sobre todo en aquellas que valoran más el tiempo en el trabajo que los resultados. Adicionalmente, Burke (2001) menciona que los trabajadores que dedican más horas a trabajar son percibidos como más comprometidos con la empresa y son tomados como empleados ejemplares.

En lo relativo a la correlación de adicción al trabajo y antigüedad ( $\mathrm{r}=0.513)$, al combinarse con la variable de la escala de satisfacción laboral, y posibilidades de promoción (carga factorial 0.90), puede interpretarse que los empleados cuentan con amplias expectativas sobre su desarrollo futuro en la empresa y por tanto, pueden pensar que entrar en sintonía con las prácticas de la organización -estilo de liderazgo jerárquico e imitar conductas-, podría ser su pasaporte para crecer en la compañía; es decir, los empleados buscan vincularse completamente con la organización con la esperanza de que trabajando como lo pide la empresa, a la larga progresarán en la empresa. Un hallazgo de esta investigación vinculado con la esperanza de progreso y antigüedad, fue que los adictos al trabajo con mayor jerarquía -directores y gerentes-, son los que tienen mayor antigüedad en la compañía y esta situación puede hacer pensar a los empleados que la perseverancia en la compañía y adoptar sin objeción la cultura de ésta, además de imitar comportamientos de las personas clave de la organizacion, redundará positivamente en su desarrollo profesional. Esta condición definitivamente puede estimular la práctica de conductas adictivas al trabajo. La sintonía entre jerarquía y esperanza de crecimiento profesional fue estudiada por Andreassen, Ursin, Eriksen y Pallesen (2012), e indican que las posiciones directivas y gerenciales están ligadas íntimamente con el narcisismo, ya que pertenecer a estos niveles implica un ascenso en la escala social, o bien, crecimiento en la jerarquía de la empresa y constituye un motivador para que los empleados de mayor jerarquía sean más afines a ser adictos al trabajo e influyan a sus colegas con su estilo de liderazgo.

Otro hallazgo importante en este estudio fue la relación que existe entre el nivel de adicción al trabajo y 
la satisfacción laboral. En este caso pudo identificarse que entre mayor nivel de adicción al trabajo, había una mayor satisfacción laboral general. Esto coincide con lo encontrado por Buelens y Poelmans (2004), quienes comentan que, en general, los adictos al trabajo están satisfechos laboralmente. Sin embargo, hay posturas opuestas a lo observado en este estudio, como sostienen Spence y Robbins (1992), que argumentan que un elemento característico del adicto al trabajo es la insatisfacción laboral. Por su parte, Schaufeli, Bakker, Heijden y Prins (2009), comentan que la adicción al trabajo está negativamente relacionada con varios indicadores como satisfacción en el trabajo y felicidad; de hecho, estos autores consideran la adicción al trabajo como un constructo negativo e ignoran este aspecto de satisfacción laboral y el enfoque de su idea es que por definición hay insatisfacción laboral en un empleado adicto al trabajo.

En este estudio, el que haya una relación positiva entre satisfacción laboral general y adicción al trabajo, pudiera estar influido por la cultura de la organización o por la cultura del país en la que opera esta empresa, por lo que habría que estudiar con mayor profundidad el impacto de estos elementos sobre la adicción al trabajo.

En cuanto al desempeño laboral, en esta investigación se encontró que a mayor nivel de adicción al trabajo hay mejor desempeño laboral. Esto contradice lo expuesto por algunos autores sobre el tema, que comentan que el adicto al trabajo tiene un pobre desempeño laboral y más bien es inefectivo; entre tales autores se encuentran: Burke (2001); Del Líbano y Llorens (2010); Fassel (1990); Robinson (1997); Schaufeli, Taris y Bakker (2006); y Spence y Robbins (1992).

¿Cuál podría ser la explicación del resultado en este estudio de caso que contradice lo encontrado por

\section{REFERENCIAS}

Andreassen, C. S., Ursin, H., Eriksen, H. R. \& Pallesen, S. (2012). The relationship of narcissism with workaholism, work engagement, and professional position. Social Behavior and Personality, 40(6), 881- 890.

Buelens, M. \& Poelmans S. A. Y. (2004). Enriching the Spence and Robbins' typology of workaholism: demographic, motivational and organizational correla- otros investigadores? Pudiera explicarse por la forma en que se concibe el desempeño laboral en la organización sujeta a estudio. Por ejemplo, el desempeño laboral en ésta se encuentra orientado al cumplimiento de objetivos, no importa la manera de cumplirlos, si ésta es eficiente o no eficiente -si se hace en una o en 48 horas de trabajo continuo, trabajando a prueba y error, sin dirección aparente-, lo que buscan es la efectividad en el objetivo -cumplirlo a costa de lo que se necesite hacer-; por ejemplo, la fecha de entrega de un proyecto, el arranque de una operación de ventas, etcétera.

Schaef y Fassel (1989) hacen mención a que el desempeño es la combinación de efectividad y eficiencia. Sin embargo, también comentan que en un sistema cerrado, correspondiente a una empresa adictiva, el paradigma vigente es el que pone las reglas y lo que no está en éste, simplemente no puede verse o no existe para la organización, por lo que en este caso la empresa sujeta a estudio valora la efectividad en el trabajo de los ejecutivos - cumplimiento de objetivos-, y quizá la eficiencia en el trabajo de éstos - eficientar tiempo y recursos-, es algo que tiene poca importancia para la empresa o no existe en su paradigma.

\section{RECOMENDACIONES PARA FUTURAS INVESTIGACIONES}

Se sugiere tomar una muestra estadísticamente más amplia y repetir el estudio para poder hacer generalizaciones estadísticas. Otra recomendación es diseñar un cuestionario para medir el impacto que tiene el medio ambiente laboral sobre la conducta de los participantes hacia el trabajo, ya que éste es un elemento que puede tener gran influencia y valdría la pena determinarlo. •

tes. Journal of Organizational Change Management, $17,440-445$.

Burke, R. J. (2001). Workaholism in organizations: the role of organizational values. Personnel Review, 30, 637-645.

Cook, C., Wall, J. P. \& Warr, W. (1981). The experience of work. Londres: Academic Press. 
Del Líbano, M. \& Llorens, S. (2010). Adicción al trabajo: guía de intervención. Madrid: Síntesis.

Del Líbano, M., Llorens, S., Salanova, M. \& Schaufeli, W. (2010). Validity of a brief workaholism scale. Psycothema, 22, 143-150.

Doerfler, M. C. \& Kramer, P. P. (1986). Workaholism: sex and sex role stereotyping among female professionals. Sex Roles, 14, 551-560.

Fassel, D. (1990). Working ourselves to death: the high costs of workaholism, the rewards of recovery. San Francisco, California: Harper Collins.

Herzberg, F. (1968). One more time: how do you motivate employees. Harvard Business Review, 68(9), 5-17.

INEGI (2012). Encuesta nacional de ocupación y empleo: conjunto de datos población ocupada. Recuperado de http://www.inegi.org.mx/lib/olap/consulta/general_ ver4/MDXQueryDatos.asp?prog=enoe2011_po

Kanai, A., Wakabayashi, M. \& Fling, S. (1996). Workaholism among employees in Japanese corporations: an examination based on the Japanese version of workaholism scales. Japanese Psychological Research, 38(4), 192-203.

Killinger, B. (1991). Workaholics: The respectable addicts. Nueva York: Simon \& Schuster.

Korn, E. R., Pratt, G. J. \& Lambrou, T. (1987). Hyperperformance: the A.I.M. strategy for releasing your business potential. Nueva York: John Wiley.

Locke, E. A. (1976). The nature and causes of job satisfaction. En M. D. Dunnette (Ed.). Handbook of Industrial and Organizational Psychology. Chicago, Illinois: Rand McNally.

Machlowitz, M. M. (1978). Determining the effects of workaholism. New Haven, Connecticut: Yale University Press.

Milkovich, G. \& Boudreau, J. (1994). Dirección y administración de recursos humanos. Nueva Jersey: Addyson Wesley Iberoamericana.
Mosier, S. K. (1983). Workaholics: an analysis of their stress of work commitment. Austin, Texas: University of Texas Press.

Oates, W. (1971). Confessions of a workaholic: the facts about work addiction. Nueva York: World Publishing.

Porter, G. (1996). Organizational impact of workaholism: suggestions for researching the negative outcomes of excessive work. Journal of Occupational Health Psychology, 1, 70-84.

Porter, G. (2006). Profiles of workaholism among high tech managers. Career Development International, 11, 440-462.

Robinson, B. E. (1997). Work addiction: implications for EAP counseling and research. Employee Assistance Quarterly, 12, 1-13.

Robinson, B. E. (1998). Spouses of workaholics: clinical implications for psychotherapy. Psychotherapy: theory, research, practice, and training, 35, 260-268.

Salanova, M., Del Líbano, M., Llorens, S., Schaufeli, W. E. \& Fidalgo, M. (2008). La adicción al trabajo: una nota técnica de prevención. Instituto de Nacional de Seguridad e Higiene en el Trabajo, 759, 22a. serie.

Schaef, A. W. \& Fassel, D. (1989). The addictive organization. San Francisco, California: Harper Row.

Schaufeli, W. B., Bakker, A. B., Van der Heijden, F. \& Prins, J. T. (2009). Workaholism among medical residents: it is the combination of working excessively and working compulsively. International Journal of Stress Management, 16, 249-272.

Schaufeli, W. B., Taris. T., W. \& Bakker, A. (2006). Dr. Jekyll and Mr. Hide. En R. J. Burke (Ed.). Research companion to working time and work addiction (193217). Northhampton, MA: Edward Elgar.

Snir, R. \& Zohar, D. (2000). Workaholism: work-addiction or workphilia? Israel: University of Haifa Press.

Spence, J. T. \& Robbins, A. S. (1992). Workaholism: definition, measurement and preliminary results. Journal of Personality Assessment, 58, 160-178. 